\title{
Legitimación cognitiva de políticas de calidad universitaria a través del proceso de aprendizaje y rendimiento académico
}

\section{Cognitive legitimation of the university's quality policy through the learning process and academic performance}

Sandra E. Rodríguez A. Coordinadora del Doctorado en Educación de la UPNFM universidadpedagogicadoctorado@gmail.com

\section{Resumen}

Existe una variedad de comportamientos estudiantiles relativos al aprendizaje, más no todos conducen a un rendimiento académico avanzado. Sin embargo, las instituciones declaran en su política, estándares de calidad a cumplir por los actores que intervienen en el hecho educativo. El problema está en la construcción de esa política y cómo se disemina y arraiga en la cognición de éstos, de manera que los procesos continuos para el aprendizaje y los resultados de éstos, sean congruentes con las expectativas declaradas por la institución, ya que su visión es posicionarse frente a sus públicos, con una imagen de calidad. Para tener una ilustración se analizó la teoría de los constructos personales de Kelly (1969), para exponer la hipótesis: que con los años los niños y jóvenes encallan acciones relacionadas con hábitos de estudio, pero en la mayoría de los casos, no conducen a la excelencia. 
Sandra E. Rodríguez A

Se planteó como objetivo de la investigación-acción: explicar los procesos de aprendizaje que emplean los universitarios y en consecuencia su rendimiento académico y a partir de los resultados, intervenir en pro de la mejora de los procesos de aprendizaje.

Palabras claves: legitimación cognitiva, política de calidad, procesos de aprendizaje, rendimiento académico.

\section{Abstract}

There is a variety of students' behaviors related to their learning, but not all lead to an advanced academic performance. However, institutions declare in their policy, quality standards to be fulfill by the involved actors within the educational process. The problem lies in the policy's construction and how to disseminate and take root in their cognition so that the continuous learning and its results be congruent with the institution's expectations, since its vision is to position itself in front of the public with a quality image. To have an overview, it was analyzed the theory of the personal construct by Kelly (1969), to expose the hypothesis: that over the years the children and the youngs aground actions; these are related to study habits, however in the majo rity of the cases not lead to excellence.The action-research method was the stated objective: explain about the learning processes which are used by the university students and consequently about their academic performance. From the obtained results, proceed with the improvement of the learning processes.T he sample comprised two groups of college students, these allowed the comparison of the past study trends and post-intervention trends.

Keywords legitimation co gnitive, po litics of educational quality, learning process, academic performance. 
Legitimación Cognitiva de Políticas de Calidad Universitaria

a través del Proceso de Aprendizaje y Rendimiento Académico

\section{Introducción}

Las universidades deben asumir su rol de generadoras de conocimiento y a través de sus unidades de inteligencia, determinar cuáles son los factores de éxito con que cuentan, cuáles tiene que alcanzar mediante un plan proactivo-sostenido y una de las estrategias es su política de calidad. Ya Fullat (1994, pág. 189), argumentaba que se requiere de libertad para su ejecución "esta es la capacidad de adherirse a la institución", Es decir,es un acto voluntario de los actores de la educación, a fin de "consolidar la formación integral mediante la aplicación de las mejores prácticas en calidad educativa, desarrollo de competencias, vinculación, investigación, emprendimiento y el uso de tecnología de vanguardia..." para lograr la sostenibilidad universitaria (www.unitec.edu/acerca/ políticas-de-calidad).

Uno de los factores que evidencia el cumplimiento de una política de calidad educativa y que hace exitosa a toda institución que ofrece este servicio, es el rendimiento académico óptimo de sus estudiantes, concebido como "el producto de los comportamientos en el que no sólo se contemplan las aptitudes y la motivación del estudiante, también hay otras variables intervinientes como los aspectos docentes, la relación profesor-estudiante, el entorno familiar y social" (García y otros, 2000, pág. 248). Éste requiere de una construcción cognitiva so bre los procesos de aprendizaje a los que hay que ajustarse, que es el objeto de este trabajo: el ambiente en que se estudia, el cuidado de su salud física y emocional, los métodos empleados, organización de los planes y horarios de estudio, cómo busca la información, cómo comunica de forma oral y por escrito, que tan motivado para aprender está el estudiante, cómo visiona su profesión y qué investiga sobre la misma.

Es relevante identificar cómo los estudiantes enfrentan su aprendizaje: "su atención, motivación, el conocimiento, las ideas preconcebidas y generativas" (W ittrock, 1992, pág. 531)1, es un buen punto de partida

\footnotetext{
${ }^{1}$ Traducción propia: (a) attention, (b) motivation, (c) knowledge and preconceptions, and (d) generation.
}

Paradigma - Revista de Investigación Educativa. Año 21. No. 35 
Sandra E. Rodríguez A

para obtener un diagnóstico educativo, que permita analizar el estado real en que se encuentra el estudiante, conocer sus capacidades e insuficiencias. También ha de considerarse que el diagnóstico permite tener una óptica para la prevención del fracaso, la deserción y a su vez el método para disminuirlo, o en el mejor de los casos, para elevar la calidad de su formación.

Los desafíos de las universidades, en este caso de Honduras, es la "acreditación de carreras de grado y postgrado... y la atención está en la docencia, pero también (se requiere que sus estudiantes demuestren capacidades para) la investigación y la vinculación con la sociedad... conlleva un reto muy grande que es preciso enfrentar" para elevar su ranking (SICAR-C SU CA (2013, pág. 2). Estas funciones universitarias demandan espacios pedagógicos para facilitar el desarrollo de capacidades de búsqueda de información y co municación; de esto surge la pregunta ¿cómo se perciben los estudiantes en estas competencias que conducen a la investigación y vinculación universitaria? ¿C uentan las universidades con un estudiantado de excelencia para alcanzar sus metas?

Las instituciones educativas se ocupan de la matrícula y promoción de sus estudiantes, esta última determinada por el rendimiento académico, como indicador de eficiencia, sin indagar, intervenir y sistematizar el cómo se aprende y si éste desafía la calidad para el desarrollo de competencias académicas profesionales. Con una discusión en agenda, que todavía no da respuesta al "por qué muchos estudiantes no se sienten satisfechos en sus estudios, ipor qué desertan de la univer sidad? por qué pasan (sus periodos académicos) con notas bajas y otros en cambio, por qué se sienten a gusto, por qué ven la universidad positivamente, se comprometen con sus estudios y con su formación" (A rtunduaga, 2008, pág. 1). Estas respuestas evitarían el abandono, que puede surgir después de algunas semanas, o meses de haber comenzado un curso y hay que darle respuesta del porqué de la ausencia: de un método de estudio aplicado, un bajo nivel de: competencias, motivación, econo mía y otras variables que pueden explicar los comportamientos de los universitarios. 
En el supuesto del bajo número de estudiantes con rendimientos óptimos, se ha investigado en este espacio, mediante la pregunta problema ¿cuáles son los comportamientos académicos referidos a los procesos de aprendizaje y rendimiento de los universitarios de la muestra? de manera que los resultados permitan valorar los antecedentes y consecuencias, de ahí, ubicarles como estudiantes de nivel avanzado y/ 0 satisfactorio o totalmente insatisfactorio y si deben mejorar sus compromisos hacia la calidad.

Para dar respuesta a la pregunta núcleo de la investigación, se propuso como objetivo: explicar sobre los procesos de aprendizaje que aplican los universitarios y en consecuencia su rendimiento académico y a partir de los resultados intervenir en pro de la mejora de la calidad educativa de estos.

\section{Metodos y Materiales}

Se planteó comprender los procesos que aplican los estudiantes mediante la investigación-acción educativa, en los tér minos citados por McKernan (1999, pág. 53), este puede ser de enfoque cuali 0 cuantitativo ${ }^{2}$, en este caso se ha estudiado desde el segundo, debido a los registros hechos en el estudio; cabe aclarar que se hizo interpretación e intervención del problema, a fin de incrementar los procesos de aprendizaje y en consecuencia el rendimiento académico, esto hace que además de tener un alcance descriptivo, explique los hallazgos debido a una intervención de diseño longitudinal de dos meses.

\footnotetext{
2 Según McKernan (1999, pág. 53), “la investigación-acción moderna, no encaja en un enfoque estable (cuanti-cuali), pero reconoce diversos estilos". Si se requiere que el profesor sea neutral y evitar de forma sistemática, conducir las opiniones por cauces preestablecidos. Elliott (1990), ha distinguido el paradigma de la investigación sobre la acción, que le denomina investigación educativa. Esta ha sido mantenida por autores posteriores que han tratado el tema de la investigación en el aula. Una de las situaciones que más ha contribuido al desarrollo de la investigación educativa, ha sido la participación de los profesores como investigadores, propugnada por Stenhouse (1987). Hay que salvaguardar la diferencia de opiniones y facilitar la libertad de los estudiantes para manifestarse. También hay que considerar que puede ser una limitación que el profesorado no pueda aplicar esta técnica, si las autoridades de dirección no están implicadas en lo mismo, pueden haber reacciones no favorables.
} 
El problema de ¿cómo aprenden los estudiantes, qué resultados obtienen, cómo mejorar los procesos de estudio, su rendimiento académico, evidencias de calidad en el rendimiento para el desarrollo sostenible universitario? demandó la aplicación de procedimientos en tres etapas: una aplicación de un pretest, la intervención de un taller y por último, la aplicación del postest, todas ellas acompañadas de la monitorización de la intervención.

En esta línea, se destacan los procedimientos de la intervención-acción relacionada con los procesos internos de aprendizaje del estudiante (cognitivos y motivacionales), así como aquellos externos, entre ellos el ambiente y sus relaciones con sus docentes. El ciclo de acción de la investigación tuvo dos momentos:

- La etapa previa: permitió comprender el contexto educativo (selección de la muestra) y así formular el propósito de indagación, verificar si era deseable el proyecto para las partes interesadas: docentes y estudiantes.

- El segundo, se hizo en seis pasos principales: recogida de datos la que incluyó la adaptación y aplicación de test, pilotajeretroalimentación, elaboración de base de datos en excel 2010 y SPSS-18, fiabilidad del test, tablas y gráficos; la nueva aplicación; análisis de los datos; entrega de los resultados a los estudiantes; ejecución e intervención de la muestra interesada en participar en la mejora, para ello se trabajó el mes de julio y agosto, 2013, con talleres de planificación, motivación y académico-profesio nales; por último, los actores involucrados en el proceso de mejora, valoraron su actuación, además se les aplicó el post test y se contrastaron los resultados de los procesos de aprendizaje: el antes y el después de la intervención para mejora.

\section{Población y Muestra}

Se trabajó con una muestra por conveniencia de dos secciones de estudiantes de licenciatura, de un centro universitario y en dos de sus campus, ubicados en Tegucigalpa: en el grupo 1, fueron 18 estudiantes y del grupo 2 fueron 33, cada uno de dos diferentes carreras: administrativas y sociales. 
Legitimación Cognitiva de Políticas de Calidad Universitaria a través del Proceso de Aprendizaje y Rendimiento Académico

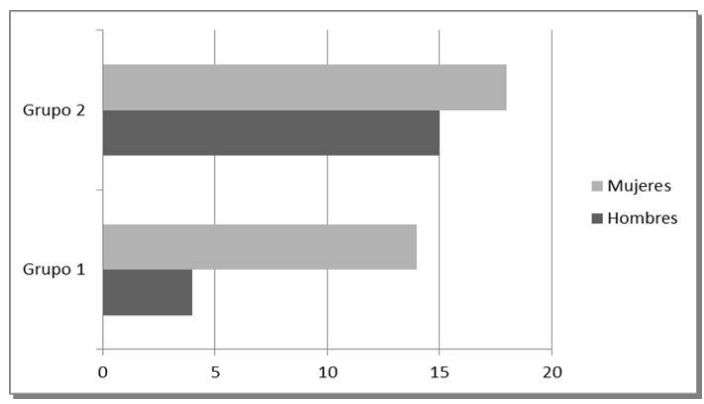

Figura 1

Género de los estudiantes

Fuente: Muestra de 18 y 33

estudiantes que participaron

en la primera fase de la investigación

\section{Instrumento}

Se utilizó el test (instrumento) derivado de Schmeck et al. (1983): inventario de procesos de aprendizaje (1991), que se ha desarrollado en el contexto de la psicología cognitiva, derivado de las teorías de procesamiento de la información (Esteban, Ruiz, \& C erezo, 1996, pág. 135). Más de 30 años avalan este instrumento desde la aparición de la primera versión en Estados U nidos. En este caso se adaptó el validado por 0 ñate (1999), y se le hizo una agrupación al inventario de prácticas de estudio en nueve secciones, con 10 ítems las primeras ocho y 20 la última, tal como se describen en la tabla siguiente.

Tabla 1. Secciones del test

\begin{tabular}{|lc|}
\hline \multicolumn{1}{|c|}{ Secciones } & N úmero de ítems \\
\hline \hline Factores ambientales & 10 \\
Salud física y emocional & 10 \\
Método de estudio & 10 \\
Organización de los planes y horarios de estudio & 10 \\
Realización de exámenes & 10 \\
Búsqueda de información & 10 \\
Comunicación oral y escrita & 10 \\
Acerca de la motivación para aprender & 10 \\
Estrategias de aprendizaje con salida profesional & 20 \\
\hline
\end{tabular}

Fuente: clasificación propia, a partir de las 100 preguntas del test aplicado.

Paradigma - Revista de Investigación Educativa. Año 21. No. 35 
El formato del test está elaborado con la escala de Likert, con cuatro alternativas de respuesta que van desde la afirmación "me describe totalmente" hasta la alternativa "no me describe en absoluto". Al final del test, se incluyó una pregunta abierta, para que el participante indicara las causas a las que atribuía su ocurrencia en los procesos de estudio y el ambiente que tenía para hacerlo.

Se analizó la fiabilidad de la prueba para la muestra de estudiantes de la U niversidad en estudio, se obtuvo un alfa de Cron Bach entre 0.6, y es explicado con un $65,76 \%$ de la varianza total, lo que es adecuado. Se utilizó el programa de SPSS 18.

Tabla 2. Fiabilidad y varianza del test

\begin{tabular}{|c|c|c|c|c|c|c|}
\hline \multicolumn{3}{|c|}{ Scale Statistics } \\
\hline Mean & Variance & $\begin{array}{c}\text { Std. } \\
\text { D eviation }\end{array}$ & Ítems & $\begin{array}{c}\text { Cronbach's } \\
\text { A lpha }\end{array}$ & $\begin{array}{c}\text { Alpha Sta } \\
\text { Items }\end{array}$ & $\begin{array}{c}\text { N of } \\
\text { Ítems }\end{array}$ \\
20,1372549 & 65,7607843 & 8,10930233 & 100 & 0,60138748 & 0,59949995 & 100 \\
\hline
\end{tabular}

Fuente: programa SPS 18 'para el análisis estadístico de los ítems.

\section{Contexto del caso de estudio y acciones de intervención}

Se trabajó en la primera fase con un grupo de jóvenes entre 18 y 22 años de edad para el primer grupo y de 23 a 34 en el segundo grupo. U bicados en dos contextos geográficos diferentes: el grupo 1 estudiaba y el grupo 2 además trabajaba.

En la segunda fase se trabajó con el consenso de los docentes y se propició una sesión de análisis para la intervención con los estudiantes. La entrega del informe a cada estudiante se hizo de forma individualizada y se envió por correo a los estudiantes que no pudieron asistir. Con el grupo interesado se desarrollaron talleres de reflexión con temas como: 10 pasos hacia el éxito académico, estilos de aprendizaje y de pensamiento, planificación del tiempo de estudio, 
búsqueda de información, comunicación escrita y temas libres. En todo el proceso se observó e indagó sobre el comportamiento de los estudiantes, sus relaciones sistémicas entre ellos, su nivel de crítica hacia sus compromisos estudiantiles, no cumplidos hasta al momento de la intervención. Estos se atendieron en el espacio físico de su aula, asimismo por la vía electrónica. Al final, se trabajó en el aula con el llenado del pos test.

\section{Análisis de resultados}

Se tomó en consideración las escalas 3 y 4 del test, que son las que ubicaron a los estudiantes en los siguientes niveles ${ }^{3}$.

- Avanzado: es el nivel correspondiente a los estudiantes que dieron respuesta a las nueve catego rías y a la mayoría de los 100 ítems, en la escala valorada en 4 y 3 , los que se ubicaron con un porcentaje entre $76 \%$ y $100 \%$ correspondió a este percentil, así mismo se cotejó con su rendimiento académico para identificar la congruencia entre su desempeño y rendimiento excepcional.

- Satisfactorio: son aquellos estudiantes que valoraron las respuestas de las nueve categorías y la mayoría de los 100 ítems, en la escala de 4 y 3 , y que se ubicaron en un porcentaje entre $51 \%$ y $75 \%$

- Debe mejorar: se situaron aquellos estudiantes que dieron respuestas mínimas a los procesos de aprendizaje que aplicaban y que no son suficientes, colocándoles entre $26-50 \%$.

- Insatisfactorio: los estudiantes en este nivel no tienen los conocimientos mínimos de los procesos que expresan los ítems de cada categoría, sobre prácticas de estudio, no son suficientes para su avance y rendimiento académico, auto ubicándose en un porcentaje menor a 25.

\footnotetext{
${ }^{3}$ Se aplicó la clasificación de la Secretaría de Educación, según el informe de rendimiento académico, 2012.
}

Paradigma - Revista de Investigación Educativa. Año 21. No. 35 
Sandra E. Rodríguez A

\section{DiscusiónTeórica}

El análisis teórico que explica los procesos de aprendizaje de los estudiantes y sus rendimientos académicos, se hace desde dos proposiciones: una relacionada con las políticas de calidad educativa como antecedente de los co mpro misos institucionales y otra que analiza el comportamiento académico de los estudiantes y las consecuencias de éstos resultados.

Se hace un preámbulo teórico con el término política como principio de todo proceder institucional, incluyendo el comportamiento académico de los estudiantes, que es fundamental en la gestión educativa. Su alineamiento puede marcar el cumplimiento de los estándares educativos comprometidos en las políticas de calidad educativa, a su vez, los resultados pueden indicar si están en la cognición de los estudiantes; ya que "de la institución depende mucho que un estudiante esté orientado profesionalmente 0 no y el nivel de orientación se refleja en los resultados académicos. El clima institucional, el compromiso de los directivos, el interés de los profesores por mejorar el rendimiento, demuestra si una institución tiene definidas políticas claras y precisas para la promoción del éxito" (Artunduaga, 2008, pág. 4).

El vocablo política es polisémico, con lo cual es fácil utilizarlo sin saber a qué significado apunta en cada uno de sus usos. Tanto "Platón (428-348) como Aristóteles (384-322) se habían servido del término política... Santo Tomás deA quino (1228) lo incluye en sus escritos (en latín); con un significado diferente también hay registros de la utilización del renacentista Machiavelli (1469) (en italiano), la buena política... es aquella que logra sus propósitos", sea una buena política o no lo sea (Fullat, 1994, pág. 49). El autor destaca la semántica del término política con tres significados:

"política-dominio, política programa de acción y el de políticahabilidad, los tres existen inexorablemente enlazados y con superioridad de la aceptación de política de dominio, los otros 
Legitimación Cognitiva de Políticas de Calidad Universitaria a través del Proceso de Aprendizaje y Rendimiento Académico

dos sirven de instrumento a la política de dominio ... una política educativa (llamase de calidad), por tanto, que se ciñera a normativas legales, que pertenece a la política-programación, constituiría un manual frívolo e insubstancial impropio de la universidad" (pág. 81).

Es conveniente retomar el lenguaje de la política de la educación, con sus respectivos lenguajes, sean estos de categoría teórica o práctica. La política educativa de calidad requiere de libertad, argumenta el mismo autor que "esta es la capacidad de adherirse a la institución... porque las reglas son aceptadas y no impuestas" (pág. 189). El educador y los estudiantes están situados entre la libertad política y la libertad moral. Estos últimos lenguajes, influidos sobremanera, por lo que implican maneras de pensar y de sentir y en consecuencia, maneras de proceder.

Realizar trayectos entre la política educativa y el tipo de educación como política para la formación de los estudiantes, establece desafíos para la educación como política, estos desafíos son diversos y se presentan como interrogantes, por cuanto más que una descripción, ameritan un esfuerzo de problematización, de acuerdo a González (2007):

- “D esafíos históricos y realizables: ¿cuál es la perspectiva de las políticas de calidad en servicios educativos?

- Desafíos epistémicos: ¿qué aptitudes son llamadas para atravesar desde la ruta epistemoló gica de la política educativa y las alternativas políticas hacia el cauce epistémico de la educación como política de la calidad?" ( pág. 108)

La llamada política educativa, según el mismo autor, está dirigida bajo la influencia de la calidad. "Ella se erige como el concepto rector de las apuestas y movilizaciones de comunidades universitarias en A mérica Latina. La calidad... se establece como concepto rector de las demandas y de las respuestas que movilizan la educación en el mundo glo balizado contemporáneo. La calidad se instituye como la razón por 
Sandra E. Rodríguez A

la cual han de movilizarse las instituciones de educación superior para rendir cuentas a la sociedad en que se inscriben" (pág. 109).

El caso es cómo se disemina una política de calidad educativa en una institución universitaria, en donde el comportamiento viene arraigado en las actitudes de los estudiantes desde los primeros niveles educativos. Estudios empíricos como el de Cuello (2012, pág. 244), han manifestado que "existe una correlación significativa entre indicadores como el de adopción y la implementación (0.53)"; asimismo evidencia que hay una concatenación entre "difusión y adopción, difusión y diseminación, diseminación y adopción (cognitiva); todos ellos fundamentados en el postulado de Fullan (2002, pág. 190), la adopción está relacionada con las estrategias, medios y calidad de la difusión y diseminación teórica-práctica" de una política de calidad educativa.

Para explicar los procesos de transición entre la gestación de la política de calidad educativa, como un conocimiento tácito institucional, se requiere elevar la política a la cognición de los actores del hecho educativo, como medio de sostenibilidad de los servicios educativos y así obtener como resultado la generación del conocimiento explícito, que promueve con hechos el desarrollo sostenible.

Desde la perspectiva anterior, no basta con formular la política de calidad, su implementación está condicionada por las construcciones personales de los actores: estudiantes y docentes. En este canal, se ha analizado el comportamiento de los estudiantes en cuanto los procesos de aprendizaje y rendimiento académico, con las explicaciones de la teoría de los constructos personales de Kelly (1969), en su posición epistemo ló gica básica del alternativismo constructivo, que "es una posición que sostiene que la realidad no se nos revela con independencia de nuestras construcciones 0 esquemas... basados en las conclusiones que hemos sacado de la experiencia pasada y sirve para anticipar el futuro" (pág. 13 y 11). La construcción de significados de aprendizaje universitarios, está sujeto al comportamiento manifiesto en sus actitudes mediante las creencias y las consecuencias del compromiso asumido en el aprendizaje a través del devenir histórico escolar. 
Legitimación Cognitiva de Políticas de Calidad Universitaria

a través del Proceso de Aprendizaje y Rendimiento Académico

Se analiza la teoría de los constructos personales para prever 0 diagnosticar hechos y así valorar la efectividad o fracasos ocurridos. D espués de este proceso de verificación, los comportamientos pueden cambiar, esto sucederá según las percepciones del entorno, o cultura de contacto en este caso del estudiante, sus metas de cambio e innovación. Sin embargo, no todas las instituciones formado ras en los niveles básicos tienen claridad de que, "entre más temprana sea la fase en la que se encalla la persona, más grave es la patología que presenta" (Feixas y N eimeyer, 1991, pág.1), es decir, las convicciones e ideas previas de qué es y cómo debe actuar un estudiante, se van entretejiendo desde las primeras experiencias escolares e inciden en su desempeño académico en la universidad.

El evento o corolario de la construcción declara que "una persona anticipa los eventos cuando construye sus reproducciones, que sería el paso que va desde la teoría a la hipótesis; es decir, desde el sistema de construcción (conocimiento, comprensión) a la anticipación (Lobo, 2010, pág. 1). El estudiante ha venido observando que fue promovido en sus grados básicos, conforme se fue desempeñando, de igual forma lo hará en la universidad, aún cuando su promoción la haya logrado como estudiante medianamente satisfactorio y en algunos casos de forma insuficiente. Éste utiliza constructos personales aun cuando no sean ló gicos, pero le han guiado en sus acciones, válidos o no (Moreno, 1985, pág. 61), con la aclaración, de que pueden ser reformulados por el estudiante, si hay una intervención que les modele hacia un nuevo actuar académico.

Se han realizado muchos estudios como el de Ketelle (1983), que explica que el nivel global de éxito en la secundaria, es un indicador positivo del rendimiento en los estudios universitarios, es que los procesos de aprendizaje de los primeros años escolares pueden predecir el éxito o fracaso posterior. Los estudios de Toca y Tourón (1989), como los de Herrera y otros (1999), con "estudiantes universitarios, encontraron que el rendimiento académico previo, es la variable con mayor capacidad predictiva del rendimiento futuro", también concluyeron que el rendimiento anterior, es el mejor "predic-

Paradigma - Revista de Investigación Educativa. Año 21. No. 35 
Sandra E. Rodríguez A

tor del puntaje de rendimiento en la universidad" (Artunduaga, 2008, pág. 8). N o obstante, esta no solo debe absorber las prácticas que ya traen sus estudiantes, también debe incrementar los buenos procesos para el estudio.

Si la universidad se convierte en un referente de lo que es posible lograr y transmitir, con direcciones específicas so bre los compromisos de los estudiantes, para alcanzar los niveles avanzados de calidad que reclama el sistema interno y la imagen de sus públicos externos, "puede fijar una meta superior a lo esperable por los niveles culturales de donde proceden los estudiantes" (Mella \& 0 rtiz, 1999, pág. 76). La institución educativa deja de ser reflejo de la comunidad y pasa a simbolizar el mundo de los conocimientos y la clave inicial de la movilidad cultural ascendente, por lo tanto esta debe trabajar en la transición de la secundaria a la universidad, es decir pasar de lo cognitivo a lo cognoscitivo del nivel universitario, en pro del cumplimiento de su visión institucional a través de su política de calidad educativa.

\section{Resultados}

Se muestra la evidencia empírica de los procesos de aprendizaje que aplicaban los universitarios al momento de participar en este estudio, a fin de valorar si los resultados eran óptimos respecto a la escala de estudiante avanzado, según la clasificación descrita, de manera que mostraran la legitimación de su rol académico en cumplimiento de las políticas de calidad, encaminadas a la sostenibilidad de los servicios educativos. De igual forma, se muestran los resultados de la intervención con los estudiantes.

\section{Procesos de aprendizaje}

En este apartado se identifican las condiciones y los procesos para el aprendizaje que aplicaban los estudiantes del grupo 1 y 2 de la muestra. D e las 9 secciones que muestra la figura 2, la que tuvo mejor porcentaje de opinión del grupo 1 de estudiantes, sobre sus prácticas, fue la de motivación para aprender (80\%), seguido de estrategias de aprendizaje 
con salida profesional (72\%) y por último la de realización de exámenes (71\%). En la que menores prácticas mostraron los estudiantes, fue en la búsqueda de información (33\%).

El grupo 2, presentó opiniones similares al grupo 1, en las 9 secciones, como es la motivación para aprender (73\%), seguido de estrategias de aprendizaje con salida profesional (68\%) y por último la de realización de exámenes (66\%). Las menores prácticas de estudio fue en búsqueda de información (sólo un 36\% de ellos expresaron tenerla).

A l contrastar los resultados entre el grupo 1 y 2 , se observa una ligera diferencia en pro de mejores procesos de aprendizaje en el grupo 1. Sin embargo, ambos grupos de estudiantes requieren de un mayor aporte para el mejoramiento de sus estudios. Cabe destacar que la categoría de menor práctica por parte de los estudiantes es la búsqueda de información (33 y $36 \%$ respectivamente). D espués es la comunicación oral y escrita. A mbas categorías están muy relacionadas con el alto desempeño en investigación y la meto do lo gía de diseños de proyectos, manejo de reportes relacionados con la carrera de estudio, estas requieren de atención inmediata para incrementar las competencias orientadas a resolver problemas de la vida real (véase figura 2).

Figura 2

O pinión de los estudiantes respecto a sus procesos de aprendizaje

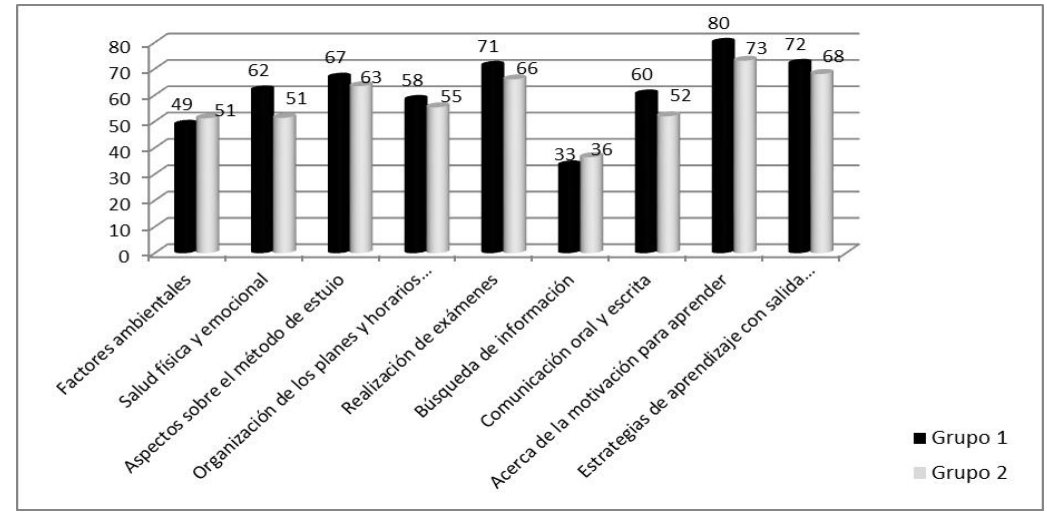

Fuente: muestra del grupo 1 y 2.

Paradigma - Revista de Investigación Educativa. Año 21. No. 35 
Sandra E. Rodríguez A

\section{Relación entre las prácticas de estudio y el rendimiento académico}

0 tro de los objetivos propuestos, fue analizar la relación entre las prácticas de estudio y el rendimiento académico de los estudiantes.AI respecto cabe destacar que las calificaciones facilitadas por los docentes de las asignaturas que cursaban los estudiantes participantes, muestran un desempeño satisfactorio, pero no hay estudiantes de excelencia académica, la mayoría está en una escala de $60-69 \%$, al igual que las prácticas de estudios, las puntuaciones tanto en la escala de excelencia académica 0 avanzada (90-100\%), como en la ubicación del percentil de prácticas de aprendizaje avanzadas, está ausente, según muestra la figura 3.

\section{Figura 3}

Índice académico de los estudiantes participantes de la investigación

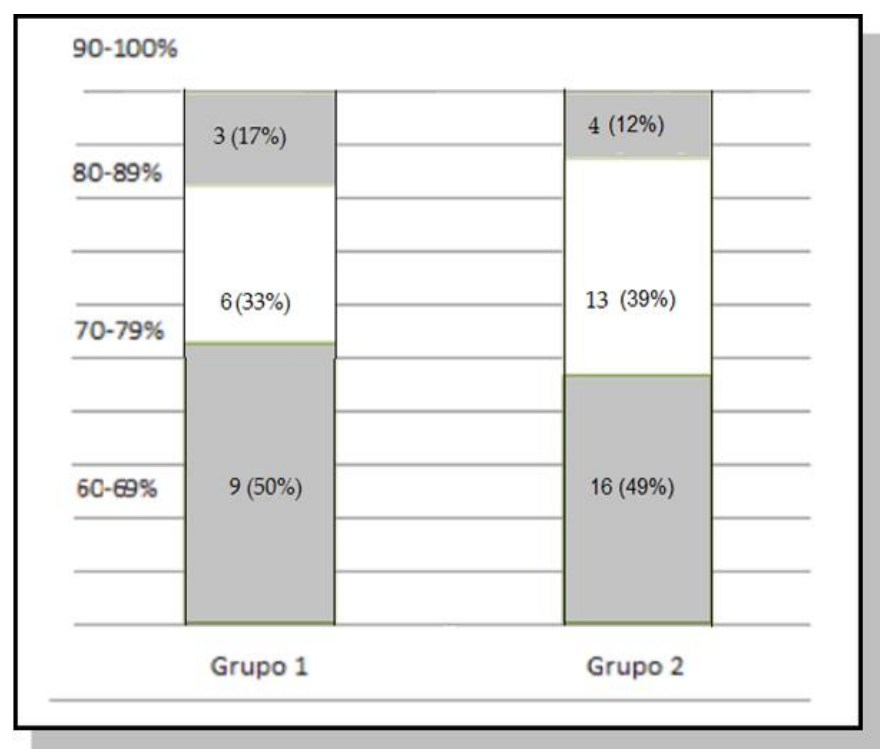

Fuente: a partir de los cuadros de calificaciones de los docentes de los dos grupos que participaron en la investigación 
Legitimación Cognitiva de Políticas de Calidad Universitaria

a través del Proceso de Aprendizaje y Rendimiento Académico

\section{Resultados de la intervención y compromisos por parte de los estudiantes}

En la segunda fase del estudio, se hizo una intervención con talleres de reforzamiento en la temática de aquellas catego rías o factores de mayor deficiencia por parte de los estudiantes, todos ellos orientados al establecimiento de nuevos compromisos: como mejorar su ambiente de estudio, ya que sus prácticas no eran saludables y cómodas, se comprometieron hacer la búsqueda de información para no copiar solamente (web), a consultar distintas fuentes y redactar sus propios informes, entre otros, esta tarea continúa en agenda.

En el nivel de las buenas prácticas de estudio, ya en la tercera fase del estudio, se manifestó una mejora según los datos comparativos entre los resultados del pretest y el postest, los que se presentan a continuación en la tabla 3.

Tabla 3. Comparación entre los resultados del pretest y el postest después de la intervención

\begin{tabular}{|c|c|c|c|c|}
\hline Grupo & \multicolumn{2}{|c|}{ Grupo 1} & \multicolumn{2}{|c|}{ Grupo 2} \\
\hline Factores & Pretes & ost test & Pretes & ost test \\
\hline Ambiente & 52 & 60 & 53 & 62 \\
\hline Salud física y emocional & 63 & 65 & 50 & 70 \\
\hline Métodos de estudio & 68 & 74 & 64 & 73 \\
\hline $\begin{array}{l}\text { O rganización de los planes } \\
\text { y horarios }\end{array}$ & 62 & 70 & 59 & 65 \\
\hline Realización de exámenes & 71 & 80 & 68 & 75 \\
\hline Búsqueda de información & 37 & 55 & 39 & 52 \\
\hline $\begin{array}{l}\text { Comunicación académica } \\
\text { oral y escrita }\end{array}$ & 65 & 73 & 55 & 65 \\
\hline $\begin{array}{l}\text { Acerca de la motivación } \\
\text { para aprender }\end{array}$ & 82 & 90 & 74 & 88 \\
\hline $\begin{array}{l}\text { Estrategias de aprendizaje } \\
\text { con salida profesional }\end{array}$ & 74 & 89 & 69 & 80 \\
\hline
\end{tabular}

Paradigma - Revista de Investigación Educativa. Año 21. No. 35 
Sandra E. Rodríguez A

\begin{tabular}{|l|l|l|l|l|}
\hline Promedio general & 64 & 73 & 59 & 70 \\
\hline
\end{tabular}

Fuente: porcentajes obtenidos con los 2 grupos de la muestra, en la primera y segunda aplicación del test

Los participantes de los talleres comentaron la necesidad de agendar por parte de la universidad, la implementación de talleres extracurriculares para el desarrollo de competencias genéricas de búsqueda de información y comunicación escrita y oral, además del sentido de pertenencia y auto concepto universitario ligado al compromiso de vida estudiantil, de cara a la excelencia académica: responsabilidad con la organización de los planes de estudio, el manejo de sus horas de ocio en pro de los hábitos que potencian la salud física y emocional. Así como un pensamiento propositivo y prospectivo de su desempeño profesional.

\section{Discusión}

Este estudio es un primer acercamiento explicativo de investigación, sobre el comportamiento de los universitarios con relación a los procesos que estos aplican para estudiar y el rendimiento académico que alcanzan, de perfil a las políticas de calidad que la institución declara, para valorar cómo actúan, deliberan, aprecian, razonan, sobre su persona, sus intereses vocacionales-profesionales, cuál es su cognición y qué transición viven, o ya han pasado hacia la legitimación cognoscitiva, es decir, ya revelan el conocimiento, las creencias y aceptación del cumplimiento de las políticas de calidad y ise refleja en sus comportamientos de estudiantes avanzados? o por el contrario, siguen en la dimensión cognitiva, sin procesar los compromisos que la universidad espera de ellos.

$H$ ay que reconocer que la legitimación cognoscitiva entre los actores universitarios responsables de la calidad académica, demanda de una construcción de la política de calidad más horizontal, su gestación corresponde a un conocimiento tácito institucional o de las autoridades que lo implantan, pero no debe quedarse ahí, requiere elevarlo a la 
Legitimación Cognitiva de Políticas de Calidad Universitaria a través del Proceso de Aprendizaje y Rendimiento Académico

cognición de los actores del hecho educativo, como medio de sostenibilidad de los servicios universitarios y así obtener como resultado la generación del conocimiento explícito en todos los actores, involucrados en el desarrollo sostenible de la universidad, conforme al prototipo visionado.

Sumado a lo anterior,el análisis del modelo teórico en esta investigación sobre los constructos personales, ayudó en la comprensión del comportamiento de los estudiantes en la universidad. Es importante el porqué de las experiencias previas de ellos; ya su hipótesis teórica plantea que cuanto más temprana sea la fase en la que se encalla la persona, más grave es la patología que presenta, esto explica los hábitos arraigados de los estudiantes universitarios, que mostraron un comportamiento preferencial por la realización de los exámenes, en detrimento de la ubicación como estudiante de nivel avanzado.

La acción razonada de los estudiantes después de la fase de intervención, es correspondiente con el corolario de la organización de la teoría de los constructos, la que señala que cada persona desarrolla un sistema de construcción que englo ba relaciones or dinales entre los constructos, convirtiéndose en una taxonomía de constructos subordinados, ya que estos no están simplemente flotando sin conexión alguna, de ahí, que se podría afirmar que si el estudiante está en contacto no verbal 0 verbal con una cultura de calidad académica, puede responder a la misma.

La efectividad de la universidad para la promoción de la mejora del rendimiento académico y actitudes de compromiso con la profesión, es cuestión de calidad percibida por sus públicos, ahora que ésta puede ser efectiva en lo académico, pero no en lo social o viceversa, o ser atractiva para la sociedad por su imagen co rpo rativa e infraestructura. Las universidades no son efectivas o infectivas en sus resultados para to dos los subgrupos que se reúnen en su interior; los efectos dependen de los constructos estudiantiles previos o estatus socioeconómico; lo que sí es puntual y posible, es el establecimiento de una ruta de calidad sostenida para la formación profesional.

Paradigma - Revista de Investigación Educativa. Año 21. No. 35 
Sandra E. Rodríguez A

\section{Conclusiones}

De las nueve catego rías relacionadas con los procesos de aprendizaje: la motivación para aprender fue la de mayor respuesta, en cambio el comportamiento de los estudiantes univer sitarios está orientado a lo inmediato, como es aprobar el examen, también hay insuficiencia de competencias en cuanto a la búsqueda de infor mación y comunicación escrita, estas son importantes para los proyectos académicos.

En los dos grupos de la muestra, se encontró que sólo un 16\% de los estudiantes manifestaron tener prácticas de desempeño entre 76-904, que corresponde al percentil de aquellos estudiantes que pueden ubicarse en avanzados y que no necesitan intervención para mejorar su aprendizaje y un $53 \%$ de ellos se ubicaron en el percentil de 51-75, que de hecho fue deseable la intervención para mejorar sus prácticas académicas. El resto de ellos, están por debajo del percentil 50 y requieren de una intervención para mejo ra de prácticas de aprendizaje de forma necesaria y urgente.

Se evidencia que la deficiencia de los procesos de aprendizaje tienen efectos considerables en el rendimiento académico, ya que tanto las calificaciones de los estudiantes como la ponderación de las prácticas de estudio, no mostraron un índice académico que les ubicara en una escala de excelencia o de estudiantes avanzados (90\%-100\%) y ambos grupos requieren de la intervención mediante un programa sostenido de calidad educativa.

Los resultados de la intervención-acción educativa, so bre los procesos de aprendizaje, mostraron un avance en el cambio de comportamiento en los estudiantes, que responde hacia el crecimiento y desarrollo académico de calidad, más alineada a la política de calidad de la universidad, según el promedio comparativo de los puntajes relacionados con las prácticas que mostraron en la primera intervención ambos grupos (61.5\% en promedio) y en la segunda, a

${ }^{4}$ Los datos mencionados en este párrafo no fueron incluidos en los resultados por espacio. 
Legitimación Cognitiva de Políticas de Calidad Universitaria

a través del Proceso de Aprendizaje y Rendimiento Académico

través de los talleres, fue de $71.5 \%$ respectivamente, lo que destaca que hay insuficiencia de actividades extracurriculares universitarias, es decir no hay espacios académicos permanentes que propicien la transición de la legitimación cognitiva a la cognoscitiva7 y responder con acierto al cumplimiento de la declaración de política universitaria en estudio: "consolidar la formación integral mediante la aplicación de las mejores prácticas en calidad educativa, desarrollo de competencias ... y sostenibilidad académica" (www.unitec.edu/política de calidad).

\section{Recomendaciones}

Los resultados invitan a hacer un análisis más fino, mediante una investigación con mayores grupos de estudiantes, a fin de estudiar la trayectoria académica, de tal manera que aunque sea válido emprender acciones globales en la institución, alineadas al modelo educativo, inclusivo del propio sistema de gestión de la calidad institucional, será necesario trazar planes específicos que incrementen la acción razonada de los estudiantes, mediante programas de rendimiento académico, proyectos entre homólogos de estudiantes, conocimientos en investigación-acción educativa; brindar apoyo, acompañamiento y orientación para que los estudiantes mejoren su calidad de vida, en las categorías de manejo ambiental, relacionada con las condiciones físicas en do nde estudian.A simismo, realizar estudios con miras a la evaluación de impacto de las acciones universitarias, frente a la percepción del público y del campo laboral, e implementar un programa de calidad educativa con temáticas de permanencia universitaria, competencias profesionales, rendimiento académico. En la misma medida, la formación docente en proyectos de investigación-acción educativa.

\footnotetext{
5 Entendida etimológicamente-cognoscere-como el desarrollo en la adquisición del conocimiento.
} 
Sandra E. Rodríguez A

\section{ReferenciasBibliográficas}

Artunduaga, M. (2008). Variables que influyen en el rendimiento académico en la universidad.

De Ketelle, J.M. (1983). Le passage de enseignement secondaire a enseignement superieur : les facteur de réussite. Humanités chrétiennes, Université Catholique de Louvain, 26 (4), 294-306.

Elliott, J. (1990). La investigación-acción en educación. Madrid: Morata. Esteban, M.; Ruiz, C., \& Cerezo, F. (1996).Validación del cuestionario ILP-R. (U. d. Murcia, Ed.) A nales de psicología, 12(2), 133-151.

Feixas, G., y N eimeyer, R. (1991). La perspectiva constructivista: Un marco integrador para la psicoterapia. Consulta 02/06/2013, hyperlinK "http://es.cyclo paedia.net/Teoria-de-los-constructos-personales-1" http://es.cyclo paedia.net.

Fullat, O . (1994). Política de la educación. Barcelona: Ediciones Ceac S.A.

García, M., Alvarado, J., \& Jiménez, a. (2000). La predicción del rendimiento académico: regresión lineal versus regresión logística. Psicothema,Vol. 12 ( Supl. N 2), 248-252.

Kelly, G .A. (1969). Psicología de los constructos personales. En Feixas, G. (Ed.). Compilador Maher, B. (2001). Barcelona:A \& M Grafic, S.L.

Mella, O \& O rtiz,I. (1999). Rendimiento escolar. Influencias diferenciales de factores externos e internos. Revista Latinoamericana de Estudios Educativos, XXIX (001), pág. 69-92.

McKernan,J.(1999). Investigación-acción y curriculum. Madrid: Ediciones Morata. 
Legitimación Cognitiva de Políticas de Calidad Universitaria a través del Proceso de Aprendizaje y Rendimiento Académico

Moreno Jiménez, B. (1985). La psicología de los constructos personales: historia, presupuestos y alcance de una teoría. Universidad Autónoma de Madrid. Estudios de Psicología, n 23-25-1985, p. 57-65.

O ñate, C. (1999). Inventario de hábitos se estudio y la mo tivación para el aprendizaje. págs. 21-42. consulta 15/07/2013. URL: http:// www.ice.upm.es/wps/cog/tutoria.

Ruiz, J. (2012). Impulsos. Consulta 20/09/2013. en http:// www.innpulsos.co m/pasos-para-desplegar-la-investigacion-accion Stenhouse, L. (1987). La investigación co mo base de la enseñanza. Madrid: Morata.

Schmeck, R.R., Geisler-Brenstein, E. y C ercy, S.P. (1991). Self-C oncept and Learning: the revised intentory of learning processes. $\mathrm{N}$ ew York: Educational Psychology, 11(3-4), 343-362.

Schmeck, R.R. (1983). Learning Styles of college students. En R.F. D illon y R.R. Schmeck (Eds.), Individual differences in cognition:Vol. I (págs. 233-279). N ew York: A cademic Press.

SICAR-CSUCA (2013). Retos y desafíos para el desarrollo de la investigación científica en las universidades del C SUCA. Primer Encuentro Bienal de Estudios de Postgrado e Investigación. Universidad de El Salvador, el 26 y 27 de octubre de 2012.

Toca,T.yTourón,J.(1989). Factores del rendimiento en los estudios de arquitectura.

Revista de Investigación Educativa.Vol. 7, N 14, 31-48.

Tourón, J. (1985). La predicción del rendimiento académico: procedimientos, resultados e implicaciones. Revista Española de Pedagogía.Año X LIII, N 169-170, 473-495. 
Sandra E. Rodríguez A

Universidad Tecnológica C entroamericana (2013).Consulta 10/08/2013, en http://www.unitec.edu/acerca/politicas-de-calidad.

W ittrock, M. C. (1992). Generative Learning Processes of the brain. C alifornia: Educational Psychologist, 27 (4), 531-541. 